\title{
Análise comparativa da distribuição dos casos graves por covid-19 no estado da Paraíba
}

\author{
Comparative analysis of the distribution of serious cases by covid-19 in the state of Paraíba \\ Análisis comparativo de la distribución de casos graves por covid-19 en el estado de Paraíba
}

\begin{abstract}
RESUMO
Objetivo: Realizar uma análise comparativa da distribuição dos casos graves por Covid-19 na Paraíba. Método: Estudo ecológico, descritivo, de série temporal, com base nas informações epidemiológicas de Covid-19 na Paraíba. Os dados foram todos os casos graves de Covid-19, no período entre a $7^{\text {a }}$ semana epidemiológica de 2020 , que registrou o primeiro caso, até a $21^{\text {a }}$ semana de 2021. Os dados foram tratados e transformados em gráficos para uma melhor análise. Resultados: Observou-se uma rápida evolução de casos graves a partir da 21 a semana epidemiológica de 2020, porém o ano encerrou-se com tendência de queda para estes casos. Após elevação em 2021, percebe-se tendência de queda ao término do primeiro semestre. Conclusão: Compreender padrões de transmissão do Covid-19, adotar medidas preventivas de controle não garantem a interrupção da disseminação da doença. A imunização contra o Covid-19, já começa revelar resultados que indicam uma possível saída da atual pandemia.

DESCRITORES: Pandemias; Infecção por Coronavírus; Epidemiologia Descritiva.
\end{abstract}

\section{ABSTRACT}

Objective: To carry out a comparative analysis of the distribution of severe cases by Covid-19 in Paraíba. Method: Ecological, descriptive, time series study, based on epidemiological information from Covid-19 in Paraíba. Data were all severe cases of Covid-19, in the period between the 7th epidemiological week of 2020, which registered the first case, until the 21st week of 2021. Data were processed and graphed for further analysis. Results: There was a rapid evolution of severe cases from the 21st epidemiological week of 2020, but the year ended with a downward trend for these cases. After an increase in 2021, a downward trend can be seen at the end of the first semester. Conclusion: Understanding Covid-19 transmission patterns, adopting preventive control measures do not guarantee the interruption of the disease's spread. Immunization against Covid-19 is already beginning to reveal results that indicate a possible exit from the current pandemic.

DESCRIPTORS: Pandemics; Coronavirus Infections; Population Density; Epidemiology Descriptive.

\section{RESUMEN}

Objetivo: Realizar un análisis comparativo de la distribución de casos graves por Covid-19 en Paraíba. Método: Estudio ecológico, descriptivo, de series de tiempo, basado en información epidemiológica del Covid-19 en Paraíba. Los datos fueron todos casos graves de Covid-19, en el período comprendido entre la séptima semana epidemiológica de 2020, que registró el primer caso, hasta la semana 21 de 2021. Los datos fueron procesados y graficados para su posterior análisis. Resultados: Hubo una rápida evolución de los casos graves a partir de la 21 semana epidemiológica de 2020, pero el año terminó con una tendencia a la baja para estos casos. Después de un aumento en 2021, se puede ver una tendencia a la baja al final del primer semestre. Conclusión: Comprender los patrones de transmisión de Covid-19, adoptar medidas de control preventivo no garantiza la interrupción de la propagación de la enfermedad. La inmunización contra Covid-19 ya está comenzando a revelar resultados que indican una posible salida de la pandemia actual.

DESCRIPTORES: Pandemias; Infecciones por Coronavirus; Densidad de Población; Epidemiología descriptiva.

RECEBIDO EM: 04/06/2021 APROVADO EM: 20/09/2021

\section{Jayne Melo Oliveira}

Graduanda em Enfermagem, UNIFACISA

ORCID: 0000-0003-4850-9263

\section{Lúcia Magnólia Albuquerque Soares de Camargo}

Professora do Curso de Enfermagem, UNIFACISA. Mestre em Saúde Coletiva. Especialista em Gestão de Serviços de Saúde e Adm. Hospitalar, Saúde do Trabalhador e Saúde Pública. Enfermeira

ORCID: 0000-0002-5442-8227 


\section{INTRODUÇÃO}

0 SARS-CoV-2, agente etiológico do coronavírus, o qual causa uma severa infecção respiratória aguda grave, teve seu primeiro caso confirmado em Wuhan, na China, no final do ano de 2019. O surto dessa doença levou a Organização Mundial de Saúde - OMS a declarar emergência na saúde pública internacionalmente, causando altos índices de mortalidade, contabilizando até o momento de elaboração deste estudo, mais de 7,9 milhões de casos no mundo, resultando em mais de 434 mil mortes, evoluindo para um estado de pandemia pelo novo coronavírus ${ }^{1}$.

O então novo coronavírus, é um vírus, o qual pertence ao subgênero Sarbecovírus da família Coronaviridae e pode ser transmitido pelo toque, gotículas de saliva eliminadas da tosse ou espirro, objetos e superfícies contaminadas. Os sintomas podem variar de assintomáticos para casos leves, moderados, graves e críticos'.

Pacientes contaminados pelo novo coronavírus podem ser assintomáticos, ou ainda apresentar sintomas leves que se manifestam de forma não específica como tosse, dor na garganta ou coriza, dor abdominal, diarreia, febre, calafrios e cefaléia. Os sintomas moderados podem incluir os sinais leves acompanhados de febre e tosse persistente. Nos casos graves os sintomas são dispnéia, desconforto respiratório ou pressão persistente no tórax, saturação de oxigênio menor que $95 \% \mathrm{em}$ ar ambiente ou cianose nos lábios e extremidades ${ }^{3}$.

No Brasil, o primeiro caso confirmado ocorreu em 25 de fevereiro de 2020 . O crescimento de pessoas contaminadas foi exponencial, e em 23 de março de 2020, o país já contabilizava 1.891 casos confirmados4. O ano de 2020 marcou o Brasil pelo seu enfrentamento a uma pandemia de rápida evolução, atingindo a marca de 1.864 .681 casos e 72.100 mortes, comprometendo cerca de $55 \%$ da população da américa latinal.

Com o número de casos alarmantes as autoridades governamentais e de saúde pública, adotaram uma série de medidas

\section{Desta forma esperava-se uma redução}

O então novo coronavírus,

é um vírus, o

qual pertence

ao subgênero

Sarbecovírus

da família

Coronaviridae

e pode ser

transmitido pelo

toque, gotículas de

saliva eliminadas

da tosse ou espirro,

objetos e superfícies

contaminadas. preventivas como, o distanciamento social, o uso de máscaras em ambientes públicos, uso de álcool a $70 \%$ para higienização das mãos, quando não for possível o uso de água e sabão5. do número e da evolução dos casos, entretanto a contaminação apenas aumentava $\mathrm{e}$ os serviços de saúde começavam a mostrar sinais de superlotação e falta de resolubilidade, exigindo do sistema de saúde brasileiro uma a ampliação dos leitos hospitalares, ventiladores e oxigênio para suporte ventilatório, educação em saúde para a população, testes rápidos, Equipamento de Proteção Individual - EPI para os profissionais de saúde, entre outras tantas necessidades de aporte médico ${ }^{6}$.

Assim, a saúde no Brasil entra em um verdadeiro colapso, o sistema de saúde passou a enfrentar, além da pandemia, outro grave problema de saúde pública, a insuficiência dos leitos nas Unidades de Terapia Intensiva - UTI. Estudos revelam que a taxa de transmissão do COVID-19, sempre em ascensão, ocorre em razão das pessoas contaminadas assintomáticas, favorecendo ciclicamente um aumento excessivo do número de casos confirmados e elevando a ocupação dos leitos de UTI no país?.

Com isso, pode-se considerar que a situação no Brasil, é extremamente preocupante, tendo em vista que o aumento da necessidade do número de leitos de UTI's, está diretamente interligado com a ascensão da curva epidêmica, que apresenta um comportamento crescente e exponencial dos casos graves do coronavírus8.

No Estado Paraíba, a pandemia pelo novo coronavírus também se alastrou rapidamente. $\mathrm{O}$ primeiro caso confirmado para Covid-19, ocorreu dia 16 de março de 2020, na cidade de João Pessoa, Capital do Estado. Rapidamente os casos confirmados aumentaram significativamente, fazendo com que o cenário no Estado acompanhasse a evolução nos demais Estados do Brasil'. Ainda sobre o Estado da Paraíba, o que chama atenção atualmente é o crescente número de casos graves, o qual chega a atingir uma média de 436 casos apenas no ano de 2021, quase o dobro da média de casos 
graves no ano de $2020^{10}$.

Neste cenário podemos observar que embora as medidas preventivas sejam conhecidas e adotadas rigorosamente os casos graves continuam crescendo a cada dia no Estado da Paraíba, e deste modo surgiu o seguinte questionamento, o qual norteou estudo: Como estão distribuídos os casos graves de Covid-19 no Estado da Paraíba? Sendo assim, o objetivo do presente estudo foi realizar uma análise comparativa da distribuição dos casos graves por Covid-19 no Estado da Paraíba.

\section{MÉTOdO}

Foi realizado um estudo ecológico, descritivo, de série temporal, com base nas informações epidemiológicas de Covid-19 no Estado da Paraíba/PB. Foram utilizados os dados graves confirmados do Covid-19 de acesso aberto do dashboard da Secretaria Estadual de Saúde. O Estado da Paraíba possui uma população estimada de 4.039.277 pessoas, distribuída em $223 \mathrm{mu}$ nicípios $^{11}$.

Os dados selecionados foram todos os casos graves confirmados para Covid-19, no Estado da Paraíba, no período compreendido entre a $7^{\mathrm{a}}$ semana epidemiológica do ano de 2020, que registrou o primeiro caso até a $21^{\text {a }}$ semana epidemiológica do ano de 2021, conforme calendário epidemiológico nacional ${ }^{12}$.

Os dados foram tratados com auxílio do programa Excel for Windows. Após tratamento dos dados, foram confeccionados gráficos para uma melhor análise comparativa dos casos graves. Não foi necessário submeter o estudo para apreciação do Comitê de Ética em Pesquisa, visto os dados serem de acesso público e sem identificação de participantes.

\section{RESULTADOS}

É possível observar a evolução dos casos graves da Covid-19 no Estado da Paraíba, a partir da $7^{\text {a }}$ semana epidemiológica do ano de 2020, tendo em vista a data do primeiro caso confirmado da doença. A Figura 1 apresenta um traçado evolutivo entre a refe-
Figura 1: Evolução dos Casos Graves - Da 7a à 11ª Semana Epidemiológica, 2020.

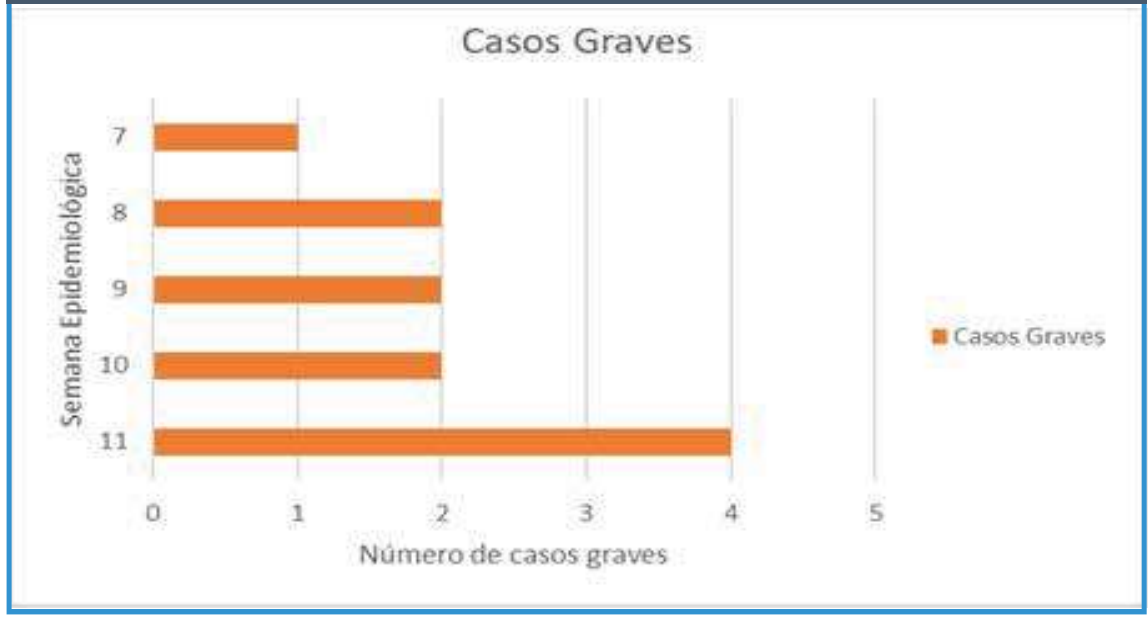

Fonte: SES/PB, 2021

Figura 2: Evolução dos Casos Graves - Da 11ª à 20a Semana Epidemiológica, 2020.

Casos do COVID-19 no Estado da Paraiba da 11 a a 202 Semana Epidemiológica 2020

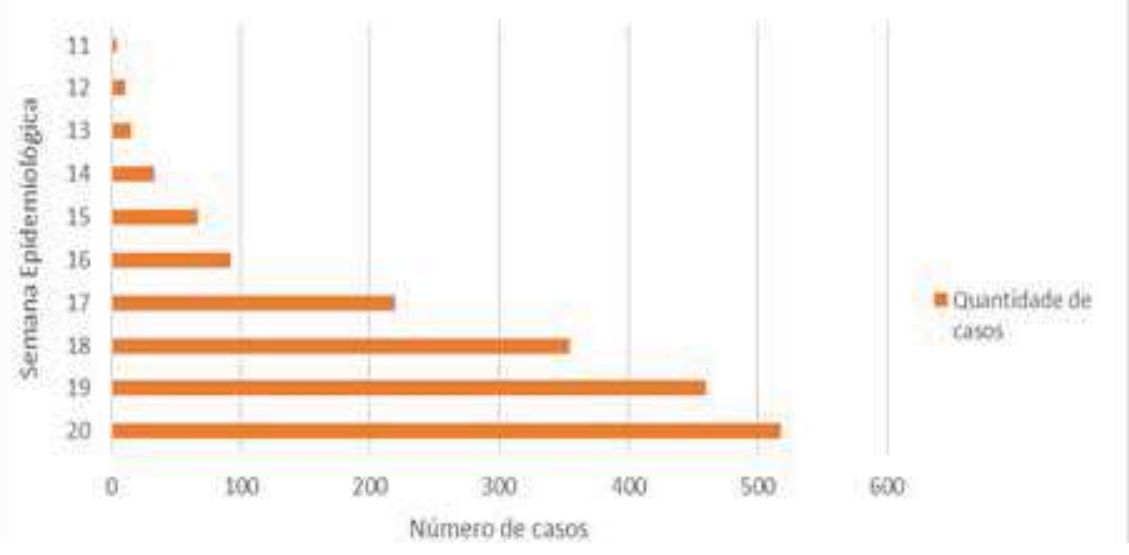

Fonte: SES/PB, 2021

rida semana até a $11^{\text {a }}$ semana epidemiológica do mesmo ano.

A Figura 2 evidencia um salto exorbitante de casos graves, é possível observar entre a $11^{\text {a }}$ a $20^{\text {a }}$ semana epidemiológica do ano de 2020, que os casos graves foram contabilizaram um total de 518 casos.

É notório que os casos graves evoluíram rapidamente, porém na Figura 3, é possível observar oscilações no número de casos, com tendência para queda de casos. $\mathrm{Na} 21^{\mathrm{a}}$ semana epidemiológica de 2020, os casos atingiram a marca de 592 casos graves. $\mathrm{Na}$ $25^{\text {a }}$ semana epidemiológica do mesmo ano (barra verde) somaram 430 casos. A tendência de queda é observada também na 26a semana epidemiológica de 2020 (barra azul) totalizando 348 casos graves. Porém, a tendência de queda é interrompida na $27^{\mathrm{a}}$ semana epidemiológica do ano de 2020 (barra amarelo) e novamente o Estado da Paraíba, volta a apresentar tendência de aumento do número de casos graves.

Ainda na Figura 3, evidencia-se certa instabilidade na evolução ou involução no número de casos graves, isto é claro ao atentar para a $30^{\mathrm{a}}$ semana epidemiológica de 2020. 
Figura 3: Evolução dos Casos Graves - Da 21ª a 30ª Semana Epidemiológica, 2020. Casos do COVID-19 no Estado da Paraiba da 312 semana a 539 semana do ano de 2020

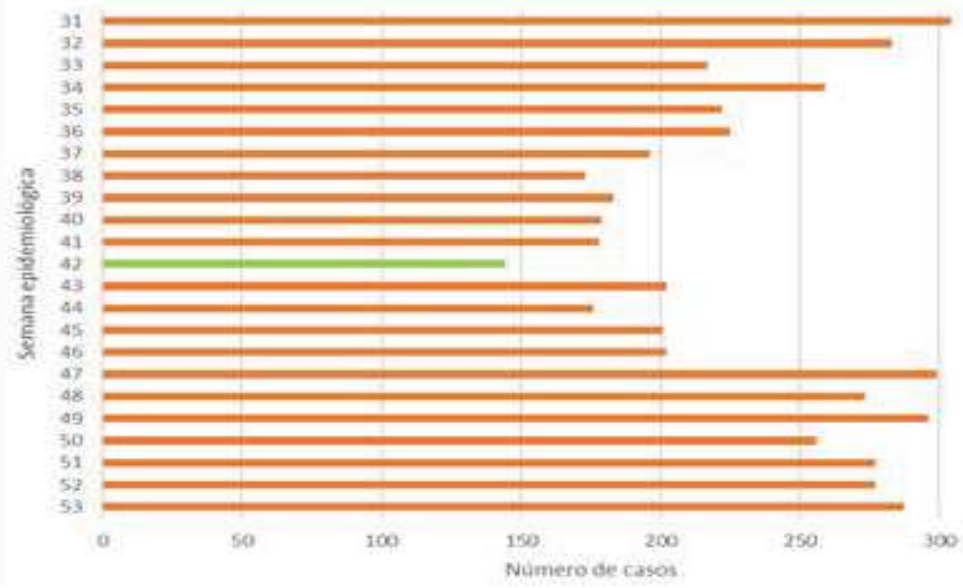

Fonte: SES/PB, 2021

Figura 4: Evolução dos Casos Graves - Da 31ª à 53ª Semana Epidemiológica, 2020.

\section{Casos do COVID-19 no Estado da Paraiba da $21^{\circ}$ a $30^{\circ}$ Semana Epidemiológica 2020}

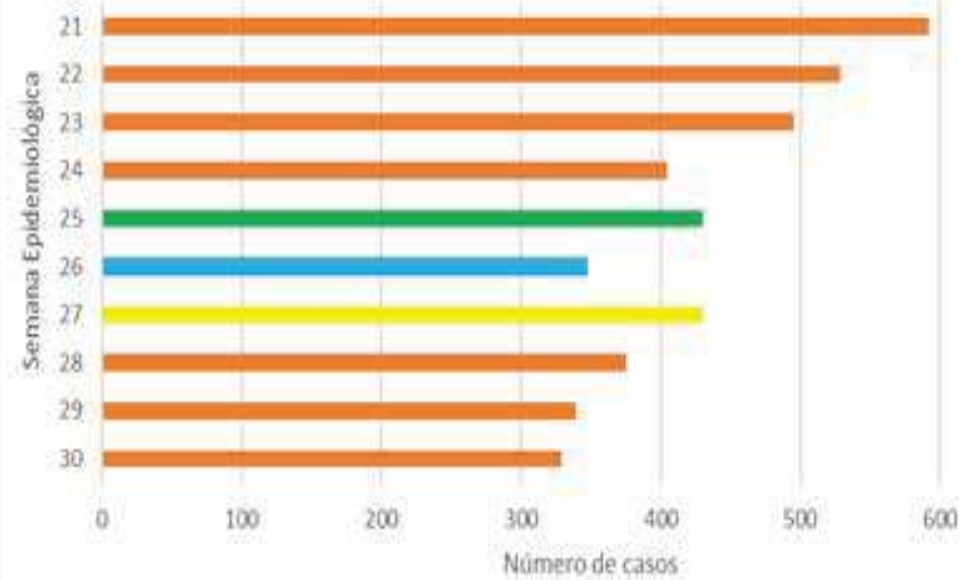

Fonte: SES/PB, 2021

A seguir na Figura 4, é possível observar uma diminuição considerável dos casos graves, no qual destaca-se a $42^{\text {a }}$ semana epidemiológica de 2020, com apenas 144 casos. Esse resultado permitiu uma transição no traçado epidemiológico no Estado da Paraíba, que mostrava-se em estabilidade, para uma tendência de queda de casos graves. Nas semanas epidemiológicas seguintes, é possível observar um aumento dos casos graves, alcançando 250 casos, e apresen- possível observar na 9a semana epidemioló gica (barra vermelha) um salto exorbitante no número de casos graves da Covid-19, comparado com as primeiras semanas epidemiológicas do ano, alcançando $829 \mathrm{ca}-$ sos. A $10^{\mathrm{a}}$ semana epidemiológica de 2021, mostra estabilidade (barra amarelo) no número de casos graves da doença, mantendo 832 casos, sendo estes, os maiores índices de casos graves no período inicial do referido ano.

Ainda conforme apresenta a Figura 5, na $15^{\text {a }}$ semana epidemiológica de 2021 , aponta uma tendência de queda do número de casos graves, com 391 casos, entretanto, na $18^{\text {a }}$ semana epidemiológica, somaram-se 522 casos. Quase como um efeito sanfona, percebe-se a partir da $20^{a}$ semana epidemiológica de 2021, uma nova tendência de queda, no qual foram contabilizados 262 casos graves da Covid-19. Até o término da coleta de dados, que compóem este estudo, na $21^{\text {a }}$ semana epidemiológica de 2021, compreendida entre 23 e 29 de maio, foram registrados apenas 50 novos casos graves para a doença.

\section{DISCUSSÃO}

De acordo com os dados apresentados no capítulo anterior pode-se observar o traçado epidemiológico do Covid-19 no Estado da Paraíba entre a $7^{\mathrm{a}}$ semana epidemiológica de 2020 e a $21^{\text {a }}$ semana epidemiológica de 2021. Foi possível observar na Figura 1 uma distribuição de apenas 04 casos graves no Estado da Paraíba, no ano de 2020. Estes casos concentraram-se nos maiores centro urbanos do Estado, sendo 02 casos em João Pessoa, capital paraibana e 02 casos em Campina Grande segunda maior cidade da Paraíba, ou seja, os casos graves, apresentados na Figura 1, estavam concentrados nas duas maiores cidades do Estado, ambas com dados demográficos de alta densidade ${ }^{11}$.

A súbita elevação dos casos graves do Covid-19 entre as $14^{\mathrm{a}}$ à $20^{\mathrm{a}}$ semana epidemiológica do ano de 2020, emergiram recomendações e determinações estaduais e municipais, emitidas pelas autoridades sanitárias a respeito do Covid-19 a fim de 
Figura 5: Evolução dos Casos Graves - Da 1ª̀ 21ª Semana Epidemiológica, 2021

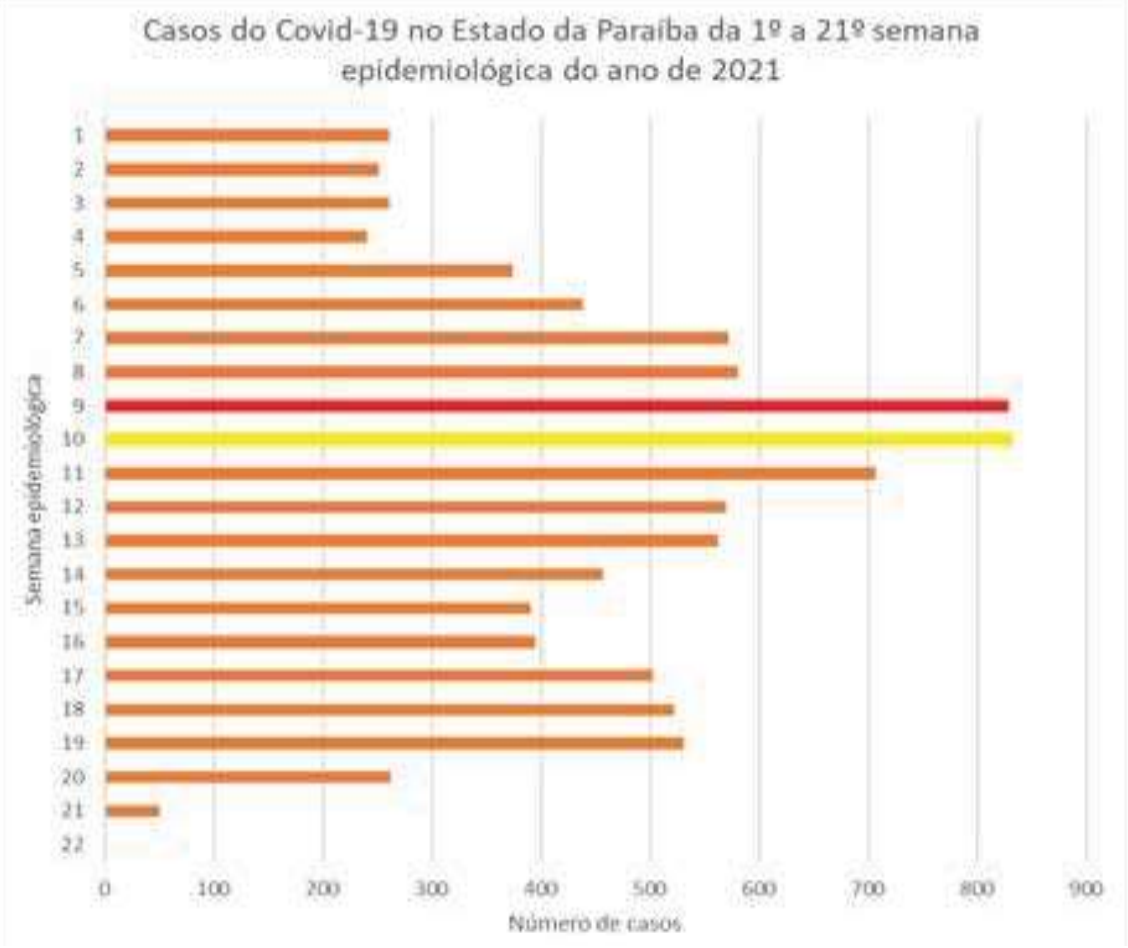

Fonte: SES/PB, 2021

conter tal avanço, através medidas mais severas, como o uso obrigatório de máscaras, lockdown, distanciamento social e testes rápidos para um controle dos casos novos13.

Apesar dos vários decretos estaduais $\mathrm{e}$ municipais, com medidas muitas vezes bastante restritivas, o número de casos graves mantinha a tendência de aumento, conforme ilustrou a Figura 3, a qual apresentou a $21^{\text {a }}$ semana epidemiológica do ano de 2021, que contabilizou mais de 500 casos graves. Os decretos restritivos no Estado da Paraíba, apresentavam seus resultados nas semanas epidemiológicas seguintes. Os casos tendiam a aumentar quando os decretos menos restritivos eram então publicados, esse resultado pode ser observado ao longo da $25^{\text {a }}$ à $30^{\mathrm{a}}$ semana epidemiológica do ano de 2020 .

É evidente que as autoridades governamentais brasileiras e de saúde pública, não estavam preparadas suficientemente para atuar no combate da pandemia do Covid-19. Parece ter faltado uma tentativa mais eficiente para que os frear o rápido aumento no número de casos e consequentemente de casos graves, tendo em vista que as medidas preventivas propostas não estavam sendo eficazes para que os casos fossem cessados. Além disso, houve demora para atentar para a sobrecarregar as redes hospitalares e falta de insumos para assistência de saúde no combate a pandemia ${ }^{14,15}$.

$\mathrm{O}$ ano de 2020 foi encerrado com tendência de queda. 2021 iniciou-se com esta tendência de queda até a $7^{\mathrm{a}}$ semana epide- miológica, porém a esperança de dias melhores com o número de casos graves para Covid-19 controlados, durou apenas até a $8^{\mathrm{a}}$ semana epidemiológica, e foi atingiu seu ápice entre $9^{\mathrm{a}}$ e a $10^{\mathrm{a}}$ semana epidemiológica, com mais 800 casos graves da doença, caracterizando o pico da doença no Estado.

O primeiro semestre de 2021 está sendo encerrado com uma tendência de queda. A imunização contra o Covid-19 no Estado da Paraíba teve início em janeiro, o plano de vacinação foi dividido por grupos de prioridade, de acordo com o tempo de exposição e pessoas com maior risco de desenvolver complicações e óbito pela doença. Até a data da coleta de dados para este estudo, a Paraíba havia recebido 368.898 doses das vacinas Coronavac e Oxford/Astrazeneca, deste quantitativo foram aplicadas 262.629, referentes à primeira dose. Hoje há 106.269 paraibanos completamente imunizados ${ }^{16}$.

\section{CONCLUSÃO}

O estudo objetivou realizar uma análise comparativa da distribuição dos casos graves por Covid-19 no Estado da Paraíba, entre os anos de 2020 e 2021, até o período proposto para a coleta de dados. Com a análise, foi possível observar que a pandemia do Covid-19 no Estado ainda não está controlada, apesar de em diversos momentos ter apresentado tendência de queda no número de casos graves.

Compreender os padrões de transmissão do Covid-19, adotar medidas preventivas de controle da doença parecem não garantir a interrupção da disseminação da doença ou melhorar o cenário no qual estamos vivendo, entretanto, a imunização contra o Covid-19, já começa revelar resultados que indicam uma possível saída da atual pandemia.

\section{REFERÊNCIAS}

1. Souza MW, Buss LF, Candido S D, Carrera JP, Li S, Zarebski EA et.al. Epidemiological and clinical characteristics of the COVID-19 epidemic in Brazil. Nature Humam Benhaviour [internet]. 2020 mar [acesso em 23 de abr. de 2021]; 04(4); 856-865. Disponivel em: https:// www.nature.com/articles/s41562-020-0928-4.

2. Lana, Raquel Martins et al. Emergência do novo coronavírus (SARS-CoV-2) e o papel de uma vigilância nacional em saúde opor- 


\section{artigo}

Oliveira, J. M., Camargo, L. M. A. S.

Análise comparativa da distribuição dos casos graves por covid-19 no estado da Paraíba

\section{REFERÊNCIAS}

tuna e efetiva. Cadernos de Saúde Pública [online]. 2020, v. 36, n. 3 [acesso em 04 de mai. 2021] , e00019620. Disponivel em: <https:// doi.org/10.1590/0102-311X00019620>. Epub 13 Mar 2020. ISSN 1678-4464.

3. Coronavírus Brasil. Disponivel em: https://covid.saude.gov.br/. Acesso em: 30 de abril de 2021.

4. Antunes PBB, Peres TI, Baião AF, Ranzane TO, Bastos LSL, Silva BAA, et.al. Progressão dos casos confirmados de COVID-19 após implantação de medidas de controle. Rev Bras Ter intensiva. 2020 mar-abr [acesso em 23 de abr. 2021]; 32(2); 213-223. Disponivel em:https://www.scielo.br/scielo.php?pid=S0103-507X2020000200 213\&script=sci_arttext.

5. Rex EF, Borges SAC, Käfer SP. Spatial analysis of the COVID-19 distribution pattern in São Paulo State, Brazil. Revista Ciência e Saúde Coletiva. 2020 ago [acesso em 24 e abr de 2021]; 25(9); 3377-3384. Disponivel em: https://www.scielosp.org/article/ csc/2020.v25n9/3377-3384/.

6. Oliveira, Wanderson Kleber de et al. Como o Brasil pode deter a COVID-19. Epidemiologia e Serviços de Saúde [online]. 2020, v. 29, n. 2 [acesso 4 de mai 2021] , e2020044. Disponivel em: <https://doi.org/10.5123/S1679-49742020000200023>. Epub 27 Abr 2020. ISSN 2237-9622. https://doi.org/10.5123/S167949742020000200023..

7. Seixas TC, Merhy EE, Feuerwerker MCL, Espirito Santo TR. A crise como potência: os cuidados de proximidade e a epidemia pela Covid-19. Interface [internet]. $2021 \mathrm{mar}$ [acesso em 24 de abr. de 2021]; 25(1); 1-15. Disponivel em: https://www.scielo.br/scielo. php?script=sci_arttext\&pid=S14143283202100020020.

8. Cavalcante, João Roberto et al. COVID-19 no Brasil: evolução da epidemia até a semana epidemiológica 20 de 2020. Epidemiologia e Serviços de Saúde [online]. 2020, v. 29, n. 4 [acesso em 04 de mai 2021] , e2020376. Disponivel em: <https://doi.org/10.5123/S167949742020000400010>. Epub 10 Ago 2020. ISSN 2237-9622. https://doi.org/10.5123/S1679-49742020000400010.
9. Secretaria Estadual de Saúde da Paraíba. Disponivel em: https:// paraiba.pb.gov.br/diretas/saude/coronavirus. Acesso em: 15 de abril de 2021.

10. Dados Epidemiológicos Covid-19 Paraíba. Disponível em: https:// superset.plataformatarget.com.br/superset/dashboard/55/. Acesso em: 30 de abril de 2021.

11. IBGE-Cidades. Disponivel em: https://cidades.ibge.gov.br/brasil/ pb/panorama. Acesso em: 26 de abril de 2021.

12. Sistema de Informação de Agravos de Notificação (SINAN). [acesso em 03 de jun. 2021]. Disponível em: http://portalsinan.saude.gov. br/calendario-epidemiologico.

13. Nacimento, Roberta Zaninelli, et al. Meio ambiente e a sua propagação da COVID-19. Brazilian Journal of Development [internet]. 2021 jan [acesso em 26 de abr. de 2021]; v.7, n.1, p.6888-6900. Disponivel em: https://www.brazilianjournals.com/index.php/BRJD/ article/view/23376/18782

14. Rafael RMR, Neto M, Carvalho BMM, David LSMH, Acioli S, Faria AGM. Epidemiologia, políticas públicas e pandemia de Covid-19: o que esperar no Brasil? Rev Enf UERJ [internet]. 2020 mar-abr [acesso em 26 de abr. 2021]; 28(e49570); 01-06. Disponivel em: https:// www.e-publicacoes.uerj.br/index.php/enfermagemuerj/article/ view/495070

15. Camargo, L.M.A.S. et al. Evolução de Indicadores e Capacidade de Atendimento Intensivo para o COVID-19 no Estado da Paraíba, 2020 [internet]. 2020 ago [acesso em 28 de abr. de 2021]; v.10i, n.56, p.3352-3363. Disponivel em: https://doi.org/10.36489/saudecoletiva.2020v10i56p3352-3363.

16. Vacina Paraíba. Disponivel em: http://vacinapb.saude.gov.br. Acesso em:03 de maio de 2021. 